\section{GASTRIC AND DUODENAL INFUSION BY MEANS OF THE DUODENAL TUBE}

\section{A NEW PROCledure IN POSTOPERATIVE TREATMENT *}

\section{GOLDER L. McWHORTER, S.B., M.D. MINNEAPOLIS}

The duodenum was first utilized for infusion in a group of dangerous biliary infections by McArthur. ${ }^{\mathrm{X}}$ Maury ${ }^{2}$ used a duodenostomy opening for this purpose in the experimental laboratory. Since Matas ${ }^{3}$ found it impossible, in some cases of cystic or of common duct obstruction, to instil fluids into the duodenum by the simple drip method through the biliary fistula, he passed a fine catheter through the biliary tract into the duodenum. It occurred to me that the passage of a catheter by mouth into the duodenum would be preferable to the biliary route, since it could be used in other than biliary fistula cases and would be more certain than the drip into the biliary drain. To the technic of a gastro-enterostomy or a stomach resection, McArthur ${ }^{4}$ has recently advocated adding the performance of a temporary biliary fistula made in the normal gallbladder.

The use of the biliary route to the duodenum in cases in which a fistula would ordinarily be made, for the treatment of some pathologic condition of that tract, would not increase the dangers of infection, of permanent fistula, or of shock from prolonging the operation; but a biliary fistula made for the purpose of duodenal infusion, in addition to some other operation, would decidedly increase those dangers, and would have a marked disadvantage over a method as simple in performance as the passage of a duodenal tube by mouth.

A review of the literature has convinced me that the use of the duodenal tube for infusion, in postoperative treatment, is a new procedure. Hemmeter and $\mathrm{Kuhns}^{\mathfrak{b}}$ were the first to construct tubes that passed the pylorus successfully. Since these tubes were somewhat bulky and complicated in construction, they did not come into general use. In 1910, Einhorn ${ }^{7}$ and Gross ${ }^{8}$ each constructed a small flexible rubber tube with a metal tip which has been successful for passage into the duodenum, and even into the jejunum.

- From the Department of Surgery, University of Minnesota Medical School.

Some Therapeutic Possibilities of Biliary Fis 1. Toure A. M. A., Jan. 1, 1910, p. 1.

2. Maury, J. D.: Discussion, McArthur (Footnote 1).

3. Matas, R.: The Gall Bladder and Biliary Tract an Avathale Therapeutic Route to the Upper Bowel, Tr. South. Surg. and Gynec. Assn., 1911, 23, 490 .

4. Gatric Surgery, Journal-Lancet, 1916, 36, No. 24.

5. Hemmeter: Arch. f. Verdauungskr., 1896, 2 (quoted by Bondi).

6. Kuhns: Arch. f. Verdauungskr., 3 (quoted by Bondi)

7. Einhorn, M.: A Practical Method of Obtaining the Duodenal Contents in Man, Med. Rec., New York, 1910, 77, 98.

8. Gross, M: A Duodenal Tube, New York Med. Jour., 1910 O1, 77; Eine Duodenalröhre, München, med. Wchnschr., 1910, 67, 1177 .
More recently Palefski $i^{0}$ showed a smaller and heavier tip to be of advantage, while $\mathrm{Hess}^{10}$ and others have employed an ordinary small catheter in infants. For use in stomach lavage, Kanavel ${ }^{11}$ has added a wire carrier to aid the passage of this type of tube.

The duodenal tube up to this time has been used for diagnosis and for therapy in medical cases. It has been used to collect material in disease of the duodenum, the pancreas and the biliary tract, to determine the degree of hemolysis, and to obtain cultures for typhoid. ${ }^{12}$ Gross $^{18}$ used direct lavage of the duodenum in chronic catarrh, in persistent jaundice and in gallstone colic, with good results. Skaller' used various medications applied directly to the duodenum. Einhorn $^{15}$ and others early used the tube for duodenal feeding in gastric and duodenal ulcer. Medical men have found feeding and medication through the tube to be of value in persistent vomiting, due to pregnancy, nervousness, stenosis and pylorospasm (in infants, Hess $^{10}$ ); in stomach disease, due to ulcer, atony, chronic dilatation, and ptosis; and in cachectic conditions, due to pernicious ancmia, tuberculosis and chronic fevers. Einhorn ${ }^{10}$ states that in cirrhosis of 
the liver duodenal alimentation contributes greatly toward diminution of the functional work of the liver.

The drip method of duodenal infusion was first described by McArthur ${ }^{4}$ through the use of a drainage opening in the biliary tract. Between feedings in cases of ulcer and persistent vomiting, Einhorn ${ }^{10}$ gave water by the drip method through the duodenal tube. Lazarus $^{17}$ has given fluids in this manner for nourishment in ulcer cases, cachectic conditions, and in some cases preparatory to stomach operations. He has used the tube a few times after operations on the stomach, but only for the purpose of preventing postanesthetic vomiting. In feeding cases, the injection into the duodenum of even moderate quantities of fluid at times causes distress, and emphasizes the importance of the drip method (Morgan, ${ }^{18}$ Jones $^{10}$ ).

Einhorn and Rosenbloom ${ }^{20}$ have demonstrated the efficiency of the duodenal route as regards absorption. They determined that there was a maintenance of the nitrogen equilibrium in patients fed by. the tube over long periods of time. Gross and Held ${ }^{21}$ state that water and salts are readily absorbed in the duodenum, and that, of the carbohydrates, the monosaccharids are readily absorbed in solutions of 6 to 8 per cent., glucose and galactose being more quickly absorbed than levulose and mannose. (Lactose may cause diarrhea.)

The duodenal tube may be passed before operation, to improve the patient's condition more readily, or during or after operation. The tube is of value as soon as it is in the stomach, since the stomach may first be washed to prevent postanesthetic vomiting; the slow infusion avoids irritation due to swallowing, and it may also be used for gavage, in persistent vomiting. In addition, the passage of the tube through the pylorus may relax it as it does in persistent vomiting due to pylorospasm $\left(\mathrm{Hess}^{10}\right)$.

That the pylorus is patent soon after operations on the intestine I have been able to demonstrate (on animals) by means of bismuth, showing that there may be no measurable delay except in extensive trauma, when there may be a delay of about an hour. Immediately after a gastro-enterostomy, however, when the pylorus was spastic, bismuth passed the union into the jejunum without delay (McWhorter ${ }^{22}$ ).

17. Lazarus, P.: Dauernährung mittels der Duodenalsonde, Berl. klin. Wchnochr., 1913, 50, 1391.

18. Morgan: Duodenal Alinentations, Am. Jour. Med. Sc., 1914, 14.8, 360 .

19. Jones, C. M.: Duodenal Feeding, Surg. Gynec. and Obst., February, 1916, p. 236.

20. Einhorn and Rosenbloom: A Study of the Nitrogen Metabolism in Three Cases of Duodenal Alimentation, Am. Jour. Med. Sc., 1911, 142, 7.

21. Gross, M. H., and Held, I. W.: Duodenal Alimentation, The Journal A. M. A., Aug. 7, 1915, p. 520 .

22. McWhorter, G. L.: A New Method of Intestinal Anastomosis, with a Study of the Intestinal Movements After Its Use, Tur JournaL A. M. A., Jan. 8, 1916, p. 86 .
The small, soft tube may be passed into the stomach or duodenum at the time of operation (Fig. 1). The introduction into the stomach may be effected by first passing the tube inside of a stomach tube and passing it, then withdrawing the latter. I have been able (in the experimental laboratory) to manipulate the metal tip through the pylorus into the duodenum from outside the stomach wall. In one experiment, using a small soft tube without a metal sip, I made a small incision in the stomach wall and pushed the end of the tube into the duodenum by means of forceps. This could easily be done at the time of a stomach resection, or in a gastro-enterostomy when the tube could be placed in the jejunum. The usual container and drip apparatus are connected to the tube, into which any desired solutions may be placed (Fig. 2). The tube may be disconnected at any time for the purpose of lavage or of aspirating, to determine the absorption. Mc $\wedge$ rthu1 ${ }^{-4}$ says that the Murphy drip or Kanavel's continuous hypodermoclysis does not accomplish the same results, since in hypodermoclysis the change in the splanchnic circulation is only seconclarily affected, and in colonic instillation the improvement in the portal circulation is not so marked as when the same fluid liathes the jejunal lining.

\section{REPORT OF CASES}

CASE 1.-D. E. M., aged 52, on whom a resection of the stomach had been performed, with a posterior gastrojejunostomy for carcinoma, received the rectal drip but had difficulty in retention. The patient vomited small amounts repeatedly for three days following the operation, totaling 450,650 and 450 c.c. daily. At the beginning of the fourth day I passed a tube by mouth. Following this, the stomach was washed and the drip started. The vomiting stopped immediately. The patient received 2,450 c.c. of sterile 6 per cent. glucose solution the following twenty-four hours. Then, after consultation with Dr. Leonard G. Rowntree, more nourishment was added in the form of 1 ounce of cream and the albumin of one egg, twice a day, and the solution was made up with 0.4 per cent. salt solution and 6 per cent. glucose with $4 \mathrm{gm}$. of sodium bicarbonate daily. The patient absorbed 1,880 c.c. the next twenty-four hours, as it seemed better to restrict the amount of liquid, and 1,550 c.c. the third. During the drip, we aspirated every two hours but could not obtain more than a few cubic centimeters at any time. At this time the patient's condition seemed so much improved that the tube was withdrawn.

CASE 2.-Mrs. M., with an acute attack of appendicitis, had vomited three or four times daily for a week. The first twenty-four hours after operation she absorbed 1,200 c.c. of fluids through the tube, and voided 500 c.c. of urine; in the second twenty-four hours she received 1,200 c.c., and voided 1,050 c.c., when the drip was discontinued because the condition was good.

In an animal experiment for another purpose, resulting in a general suppurative peritonitis following a perforation of the cecum, I inserted the tube into the duodenum. The dog (weighing 20 pounds) absorbed 1,200 c.c. of physiologic sodium chlorid solution with epinephrin the first seven hours. 
ADVANTAGE OF THE METHOD

Infusion through the permanent tube by mouth is desirable in the treatment of postoperative cases, with early nourishment and medication, since it may be instituted at any time without adding to the dangers of operation. In dehydration, acidosis, ileus and especially in the persistent vomiting following gastrointestinal operations, this method has proved of definite value.

\section{RECONSTRUCTION OF THE PYLORUS AND PYLORIC ANTRUM \\ BY TWO SUPERIMPOSED FASCIAL TRANSPLANTS AFter EXCISION OF PYLORIC ULCER *}

\section{ALFRED A. STRAUSS, M.D. CHICAGO}

The surgical treatment of gastric and duodenal ulcer has changed very little in the last thirty years. Early in that era a number of men performed both anterior and posterior gastro-enterostomy, and this has been

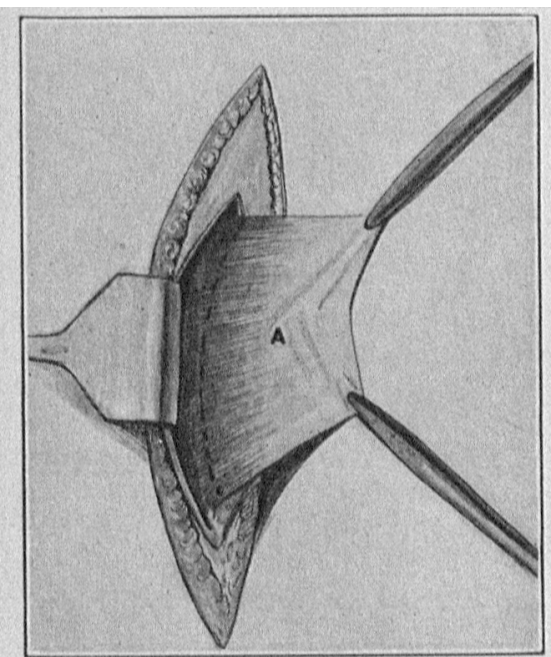

Fig. 1,-Transplant 2: $A$, transplant consisting of peritoneum, and transversalis fascia and muscle. the popular and usual operation for all forms of gastric and duodenal ulcers. When this operation was first employed, surgeons performed it because they believed it produced drainage from the stomach to such an extent that all the food would go through this opening and none through its normal pyloric opening. Since that time $m$ a n y advances have been made in the knowledge of the physiology of the stomach and also the pathology of this condition through fluoroscopic and Roentgen-ray examinations. In all these studies it has been proved that the stomach with a gastroenterostomy functionates in an entirely different manner from that for which the original operation was performed. In spite of this fact, little has been done in the line of surgery to change the surgery of the stomach in accordance with the new scientific and physiologic facts which have been demonstrated. The result is that gastro-enterostomy alone seems to be the popular operation today, and the only changes which have been made relate to its location and a few fine technical details as to suture material and type of stitch used. In simple gastro-enterostomy without pylorectomy and closure, the Roentgen ray has shown that at least 25 per cent. of food and secretion passes by the old route, thus invalidating the purpose of the operation.

About seventeen years ago, various surgeons added to this operation the closing off of the ulcer distal to

- From the Morris Institute of Medical Research of the Michael Reese Hospital. the gastro-enterostomy by what is known as a pyloric closure. The majority of the methods have not proved successful, because they were only temporary closures and for this reason did not give the desired results.

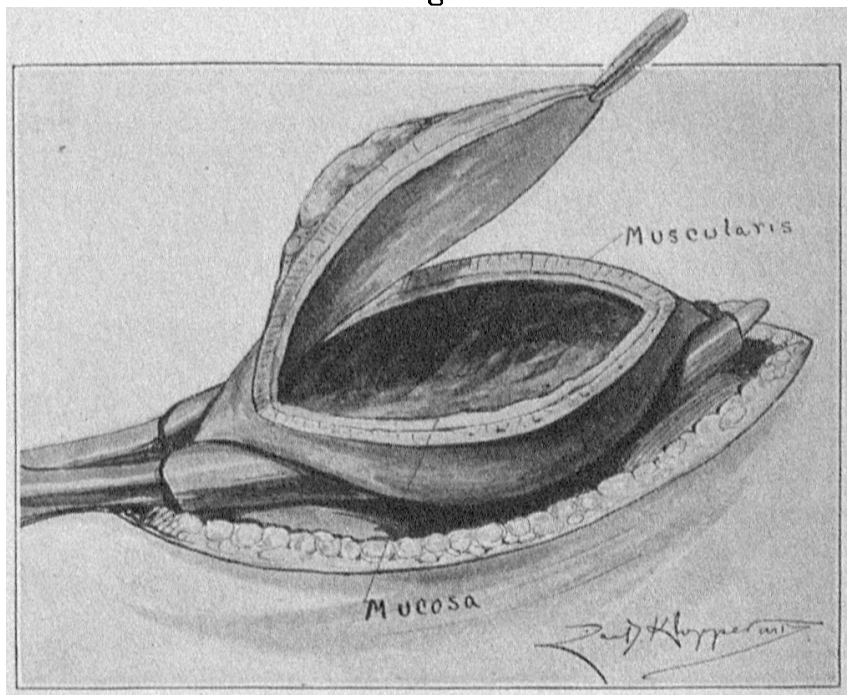

Fig. 2.-First method: application of stomach clamp and excision of ulcer.

In $1914^{1}$ I demonstrated a method of pyloric closure by using a submucous fascial transplant, which has proved experimentally and clinically to be absolutely permanent. I believe the permanent closure is a decided step forward, because it produces in the region of the ulcer all of those conditions which tend to cure an ulcer, namely:

1. By shutting off the gastric secretions and food from the stomach and duodenum, in which these ulcers occur, all irritation is arrested.

2. By using the submucous fascial transplants, a paresis is produced in the pylorus and duodenum from the point of closure around to the gastro-enterostomy, which places the ulcer at absolute rest.

I have been much impressed by the statement of medical men and surgeons that the patients who have their ulcers excised do best and have fewer symptoms

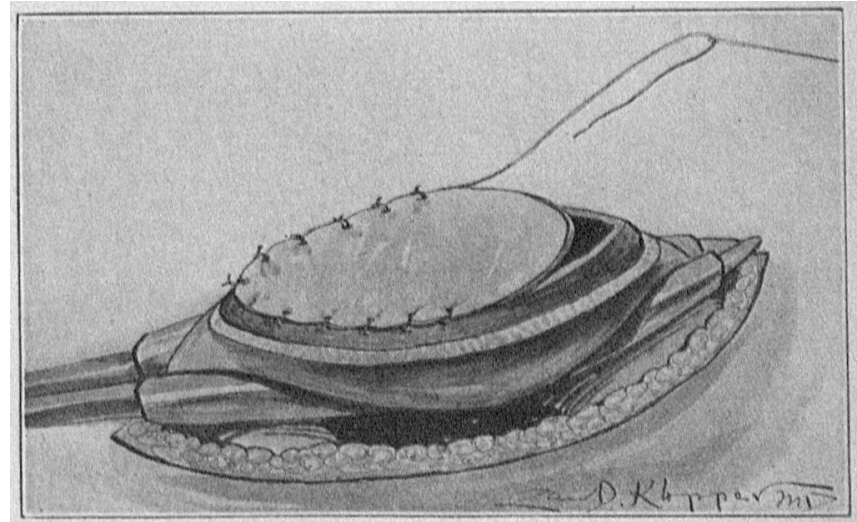

Fig. 3.-First method: suturing Transplant 2 to mucosa.

following operation, while those who have a gastroenterostomy, with or without pyloric closure, have a more protracted course of symptoms after operation. The only reason that ulcers in the pyloric region are not excised so frequently as those in the body of the

1. Strauss, A. A.: New Methods of Pyloroplasty for Congenital Pyloric Stenosis, Tur Journal A. M. A., Oct. 30, 1915, p. 1533. 